Interactive comment on "Black carbon modelling in urban areas: investigating the influence of resuspension and non-exhaust emissions in streets using the Street-in-Grid (SinG) model" by Lya Lugon et al.

Lya Lugon et al.

lya.lugon@enpc.fr

Received and published: 27 August 2021

article [utf8]inputenc [a4paper, total=6in, 8in]geometry xurl comment 
Black carbon modelling in urban areas: investigating the influence of resuspension and non-exhaust emissions in streets using the Street-in-Grid (SinG) model :

\section{Answers to referees' comments}

August 27, 2021

\section{Anonymous Referee \#1}

Received and published: 16 May 2021

\subsection{General comments}

The underestimation of $\mathrm{BC}$ concentration in air quality models has been a comment issue and this work provides some perspectives that would be important to solve this problem. Those founding are important and could be of the interest of readers and scientific communities in this field. However, the languages and overall structure of the 
manuscript should be improved before publications. My comments are as follows:

\subsection{Major comments}

1. The introduction is too long and requires serious streamlining. I would suggest the author summarize those modeling literature and emission numbers using one or two tables. And only discuss the technical difference between those works. Also, to make the overall structure more clear, the authors could consider rearranging the literature part based on the following order: observations, modeling methods, model performance, gaps need to be addressed in current models.

Author response: Yes, we agree that the introduction was too long. Following your comment and also the second referee suggestion, we added a new section (Uncertainties and variability of emissions factors observed in non-exhaust emissions). This section is included after the introduction, and include the discussions about the variability observed on non-exhaust emissions in the literature.

2. The description of different simulations conducted in this study is confusing. After careful and difficult reading, I guess there are two different sets of simulations. One set is 6 simulations with Polairs3D-SinG-MUNICH model, another one is 1 simulation with Polair3D-MUNICH model. If that is the case, the author should make it more clear in Section 2. Also, the author should improve the simulation scenario description by adding key massages in Table 1, such as the model/source used for wear emission calculation.

Author response: Different modifications were made to improve clarity, following the different comments of the reviewer. It is now specified in the Section 2 (Model description) that MUNICH can be used as a stand-alone model. This enables a better link between the Model description and the Section 5 Influence of the two-way coupling 
on $B C$ concentrations in streets (as also asked in the minor comment number 17). It is also highlighted that all the simulations presented in Section 4.1 The simulations were performed using SinG (minor comment number 13), and we added in Table 1 the reference corresponding to each test (minor comment number 15).

3. The author should explain why the simulation results present in Section 5 are not evaluate with observations. Without the model performance compared, it is impossible to evaluate the necessity or advantage to adopt the two-way coupling method compares to the one-way coupling method.

Author response: As explained in the reply to the minor comment 21, both SinG and MUNICH used as a stand-alone model (MUNICH-only) result in very similar concentrations at Boulevard Alsace Lorraine, and they both respect the most strict performance criteria. The comparisons between BC concentrations observed at Boulevard Alsace Lorraine and those calculated with SinG and MUNICH is now included. The influence of the two-way coupling depends on the street characteristics, it is more important in other streets than Boulevard Alsace Lorraine. However, there was no measurement in those streets.

Because the uncertainties of non-exhaust emissions are still high, it is difficult to evaluate with the measurements which coupling approach has better performance. As mentioned in the Conclusion (lines 500-502), "Non-exhaust emissions still present very high uncertainties, with a large spectrum of emission factors and experimental limitations to separate each non-exhaust emission source. More studies are needed to better characterize these emissions, their size distributions and chemical compositions".

4. The author takes the entire section 2 to describe a new "deposition-resuspension" framework but concludes that the whole "deposition-resuspension" process is insignifi- 
cant in improving BC underestimation (line 405-410). Instead, the selection of emission factor is the key to solve the problem. This made the work of the new "depositionresuspension" framework meanless. The author could add one more simulation with the setting of simulation 4 while turn off the new "mass-conserved depositionresuspension" framework, to properly evaluate the potential of this framework. If not, I would suggest the author move entire section 2 to the appendix and only briefly introduce the new framework in the "Simulation setup" section.

Author response: Yes, the particle resuspension calculated is this study does not strongly affect the black carbon concentration in the streets, whereas tyre-wear emissions do. This is a strong conclusion, even though it is negative (resuspension is insignificant for BC). Therefore, it seems important to accurately describe the approach used to calculate resuspension to reinforce the conclusions. Also, this approach to calculate particle resuspension is innovative and realistic, in the sense that it calculates particle resuspension as a function of the available mass on the street surface.

The simulation 5 in this study uses the setting of simulation 4, turning off the new "mass-conserved deposition-resuspension" framework. To improve clarity, the sentences

"In order to evaluate the impact of particle resuspension on $B C$ concentrations in streets, simulation 5 is similar to simulation 4, but it does not take into account particle deposition. In other words, simulation 5 simulates a maximal resuspension rate, equals to the deposition rate."

are replaced by

"In order to evaluate the impact of particle resuspension on BC concentrations in streets, simulation 5 uses the same setting of simulation 4, but it does not take into account particle deposition, and then, particle resuspension is not computed. This simulation physically represents the concentrations obtained with a maximal $B C$ resuspension, which is equal to the BC deposition.". 
5. In general, the languages used in this manuscript could be improved. Try to avoid long sentences with multiple subordinate clauses that really made the text difficult to follow.

Author response: The language was improved, and long sentences were avoided in all the modifications indicated here to answer the comments. In addition to these modifications, some sentences were also reformulated:

- At line 2, the sentence "modelling studies of BC concentrations usually underestimate $B C$ concentrations, because of uncertainties in both emissions and modelling."

is replaced by

"Modelling studies of $B C$ concentrations usually underestimate $B C$ concentrations, because of uncertainties in both emissions and modelling.".

- At line 27, the sentence

"The Parisian air-quality agency (AIRPARIF) performed a chemical speciation of particulate matter between September 2009 and September 2010, and observed that BC concentration represented approximately $4 \%$ of $P M_{2.5}$ concentrations in rural areas, $10 \%$ in urban background, reaching $27 \%$ in a street with high traffic (AIRPARIF, 2012)."

is replaced by

"The Parisian air-quality agency (AIRPARIF) performed a chemical speciation of particulate matter between September 2009 and September 2010. They found that BC concentration represented approximately $4 \%$ of $P M_{2.5}$ concentrations in rural areas, $10 \%$ in urban background, reaching $27 \%$ in a street with high traffic (AIRPARIF, 2012).".

- At line 55, the sentence

"However, Ntziachristos and Samaras (2018) also provides information about BC emissions, by providing a speciation of $P M_{2.5}$ in the form of $B C / P M_{2.5}$ ratios, which vary de- 
pending on the vehicle category (light-duty vehicles LDV or heavy-duty vehicles HDV), fuel and regulatory standard of the vehicle manufacturing (Euro norm), as presented in Table 3.91 of Ntziachristos and Samaras (2018)."

is replaced by

"However, Ntziachristos and Samaras (2018) also provide information about BC emissions, by providing a speciation of $P M_{2.5}$ in the form of $B C / P M_{2.5}$ ratios. They vary depending on the vehicle category (light-duty vehicles LDV or heavy-duty vehicles HDV), fuel and regulatory standard of the vehicle manufacturing (Euro norm), as presented in Table 3.91 of Ntziachristos and Samaras (2018).".

- At line 189, the sentence

"It investigates $(i)$ the influence of non-exhaust emissions on $B C$ concentrations in the streets, presenting a new approach to estimate particle resuspension respecting mass conservation on the street surface; and (ii) the importance of a two-way coupling between regional and local scales by comparing $B C$ concentrations in streets calculated by $\operatorname{SinG}$ and MUNICH."

is replaced by

"It investigates the influence of non-exhaust emissions on $B C$ concentrations in the streets, presenting a sensitivity analysis of wear emission factors, and a new approach to estimate particle resuspension respecting mass conservation on the street surface. It also investigates the importance of a two-way coupling between regional and local scales, by comparing BC concentrations in streets calculated by SinG and MUNICH as a stand-alone model (MUNICH-only).".

- At line 78, the sentence

"They estimated non-exhaust $P M_{10}$ emission factor to be $50 \%$ higher than the exhaust 
emission factor: $49 \%$ of $P M_{10}$ emissions were estimated to be non-exhaust emissions, $33 \%$ to be exhaust emissions and $18 \%$ were considered as unexplained emissions; brake and road wear presented an important contribution to $P M_{10}$ emissions, with almost the same emission factor as petrol exhaust."

is replaced by

"They estimated non-exhaust $P M_{10}$ emission factor to be $50 \%$ higher than the exhaust emission factor: $49 \%$ of $P M_{10}$ emissions were estimated to be non-exhaust emissions, $33 \%$ to be exhaust emissions and $18 \%$ were considered as unexplained emissions. Brake and road wear presented an important contribution to $P M_{10}$ emissions, with almost the same emission factor as petrol exhaust.".

- At line 490, the sentences

"Two main aspects influencing the $B C$ concentrations in streets are investigated: the non-exhaust emissions (tyre, brake and road wear) and particle resuspension, and the two-way coupling between the street-network model and chemistry transport model that computes the urban background concentrations. A new parametrization to calculate particle resuspension is presented, respecting the mass balance at the street surface."

are replaced by

"Two main aspects influencing the $B C$ concentrations in streets are investigated: (i) the non-exhaust emissions (tyre, brake and road wear) and particle resuspension; and (ii) the two-way coupling between the street-network model and chemistry transport model that computes the urban background concentrations. A sensitivity analysis on black carbon non-exhaust emissions is performed, regarding particularly tyre-wear emissions. A new parametrization to calculate particle resuspension is also presented, respecting the mass balance at the street surface.". 
- At line 507, the sentence

"The two-way coupling rather than one-way coupling between the local and regional scales proved to strongly influence the $B C$ concentrations in streets, because the gradients of concentrations between the local-scale street concentrations and the regionalscale background concentrations are often high."

is replaced by

"The two-way coupling rather than one-way coupling between the local and regional scales proved to strongly influence the $B C$ concentrations in streets. This is because the $B C$ concentration gradients observed between the streets (local-scale) and the urban background (regional-scale) are often high.".

\subsection{Minor comments}

1. Line 7-9, the statement after "i.e." is confusing, please rephrase. Do you mean the $\mathrm{BC}$ from one street can be transported to another street at regional scales?

Author response: We mean that the high concentrations of $\mathrm{BC}$ in a street can influence the urban background concentration above that street.

To improve clarity, the sentence

"In terms of modelling, the street models do not always consider the two-way interactions between the local and regional scales, i.e. the influence of the high $B C$ concentrations observed in streets on the urban background concentrations, which can enhance the $B C$ concentrations in streets."

is replaced by

Printer-friendly version

"In terms of modelling, the street models do not always consider the two-way inter- 
actions between the local and regional scales. With the two-way modeling approach, a street with high $B C$ concentrations may influence urban background concentrations above that street, which can finally enhance the BC concentrations in the same street.".

2. Line 22-23: Please rephrase. For example, Here comparisons are performed using ... factors from literature, and we found ?? are improved?

\section{Author response: The sentences}

"Here, emission factors of tyre, brake and road wear are calculated based on the literature, and a sensitivity analysis of these emission factors on BC concentrations in streets is performed. The model to measurement comparison shows that tyre-emission factors usually used in Europe are probably under-estimated, and tyre-emission factors coherent with some studies of the literature and the comparison performed here are proposed."

are replaced by

"Here, a sensitivity analysis of BC concentrations is performed by comparing simulations with different emission factors of tyre, brake and road wear. The different emission factors considered here are estimated based on the literature. We found a satisfying model-to-measurement comparison using high tyre-wear emission factors, which may indicate that tyre-emission factors usually used in Europe are probably underestimated. " .

3. Line 58: Can you provide the LDV and HDV definition (citation?), why the MDV (mid-duty vehicles) is not considered in this study?

Author response: In the available traffic flow observations performed during the Trafipollu campaign, and used in this study, the vehicles are classified as HDV and 
LDV. The same classification is used in other European studies, as in the NORTRIP model, defining non-exhaust emission factors only for HDV and LDV vehicle categories. HDV and LDV are classified according to the European Emission guidelines (EMEP), with light commercial vehicles lighter than 3.5 tons, and heavy-duty vehicles, heavier than 3.5 tons. Differently, the US vehicle classification also contains the MDV vehicle class, with vehicles heavier than 4.25 tons and lighter than 5 tons.

To improve clarity, the sentence

"In Europe, exhaust emission factors are nowadays determined according to the vehicle technology and fuel, providing realistic emission factors for divers vehicle fleet, as detailed in the EMEP guidelines (Ntziachristos and Samaras, 2018)."

is replaced by:

"In Europe, exhaust emission factors are nowadays determined according to the vehicle technology and fuel, providing realistic emission factors for divers vehicle fleet. The details about vehicle categories, technologies and fuel are detailed in the EMEP guidelines (Ntziachristos and Samaras, 2018)."

4. Line 75-80: Feels like most of the non-exhaust important studies are $\mathrm{PM}_{10}$ focused. Can you discuss the size distribution difference between exhaust and non-exhaust emissions?

Author response: Yes, most of the non-exhaust important studies are $\mathrm{PM}_{10}$ focused. While exhaust emissions present tiny particles, lower than $1 \mu \mathrm{m}\left(\mathrm{PM}_{1}\right)$, non-exhaust emissions present, in general, coarser particles, mostly higher than $2.5 \mu \mathrm{m}\left(\mathrm{PM}_{2.5}\right)$.

The sentence

"They estimated non-exhaust emissions to be approximately of the same magnitude as exhaust emissions." 
is replaced by:

"They estimated that the mass of coarse particles $\left(P M_{10-2.5}\right)$ from non-exhaust emissions is approximately the same as the mass of fine particles $\left(P M_{2.5}\right)$ from exhaust emissions. Note that important differences are observed in their size distribution. Exhaust emissions are composed of tiny particles, with diameters lower than $1 \mu \mathrm{m}$ (Ntziachristos and Samaras, 2018). Particles from non-exhaust emissions are coarser. According to the European Emission guidelines (Ntziachristos and Samaras, 2016), 60\% of particles emitted from tyre wear are $P M_{10}, 42 \%$ are $P M_{2.5}$, and only $6 \%$ are $P M_{1}$. Particles from brake-wear emissions have lower diameters: $98 \%$ are $P M_{10}, 39 \%$ are $P M_{2.5}$, and $10 \%$ are $P M_{1}$. The coarsest particles are those from road wear: $50 \%$ are $P M_{10}, 27 \%$ are $P M_{2.5}$, and there is no $P M_{1} . "$

5. Line 84-85: Is it the same ratio for both $\mathrm{PM}_{2.5}$ and $\mathrm{PM}_{10}$ ?

Author response: No, the authors consider that exhaust emission correspond to $\mathrm{PM}_{2.5}$, while non-exhaust emissions correspond to coarse particles, between $\mathrm{PM}_{2.5}$ and $\mathrm{PM}_{10}$.

To improve clarity, the sentence

"They estimated non-exhaust emissions to be approximately of the same magnitude as exhaust emissions."

is replaced by

"They estimated that the mass of coarse particles $\left(P M_{10-2.5}\right)$ from non-exhaust emissions is approximately the same as the mass of fine particles $\left(P M_{2.5}\right)$ from exhaust emissions."

6. Line 87: How is this related to non-exhaust emission? What is the source of this 
species?

Author response: According to Khare et al. (2020), the asphalt present in the road pavement is an important source of organic compounds. In determined temperatures and solar radiation intensities, the asphalt evaporates organic compounds during its' different life cycle stages (such as storage, paving, and use).

To improve clarity, the sentence

"Khare et al. (2020) highlighted the importance of asphalt-related emissions in SOA formation, still absent from emission inventories."

is replaced by

"Khare et al. (2020) highlighted the importance of asphalt-related emissions in SOA formation, still absent from emission inventories. These emissions are dependent of the solar radiation and temperature over the asphalt surface, and are variable according to the asphalt life cycle stages (such as storage, paving, and use).".

7. Line 91: "Some models" Please provide citations. And also, I don't think even if is appropriate here, most would be a better wording.

Author response: To use a better wording and add the citations, the sentence

"Some models try to integrate vehicle operational conditions to estimate non-exhaust emission factors, even if based on empirical factors and simplified parametrizations"

is replaced to

"A few emission models try to integrate vehicle operational conditions to estimate nonexhaust emission factors, based on empirical factors and simplified parameterizations (Guevara et al., 2020, Denby et al., 2013a)". 
8. Line 92: "HERMES" Please provide a citation of this model here.

Author response: The sentence

"The HERMES model employs the non-exhaust emission factors proposed in the EMEP guidelines (Ntziachristos and Boulter, 2016), which provide PM 10 wear emission rates for passenger cars, motorcycles, LDV and HDV."

is replaced by

"The High-Elective Resolution Modelling Emission System (HERMES) model (Guevara et al, 2020) employs the non-exhaust emission factors proposed in the EMEP guidelines (Ntziachristos and Boulter, 2016), which provide $P M_{10}$ wear emission rates for passenger cars, motorcycles, LDV and HDV.".

Please, note that HERMES model reference at line 356 was updated. The article, previously in revision, is now published.

9. Line 103: Why is it supposed to be independent? Are describing the NORTRIP assumption or making general claims? Please clear, if it is the latter, you would need a citation to justify your claims.

Author response: We describe the NORTRIP model assumption. Equation 10 in Denby et al. (2013a) describes their parameterization to calculate brake wear emissions, and it is independent of the vehicle speed.

To improve clarity, the sentence

"Differently, the NORTRIP model takes into account the vehicle speed to determine $P M_{10}$ tyre and road wear emission rates, but brake-wear emissions are supposed to be independent of vehicle speed."

is replaced by 
"Differently, the NORTRIP model takes into account the vehicle speed to determine $P M_{10}$ tyre and road wear emission rates, but not for brake-wear emissions, which are assumed to be independent of vehicle speed."

10. Line 317: Why selecting those two days? Are they more representative of the general average?

Author response: Exhaust emissions were calculated in the Trafipollu project (https: //anr.fr/Projet-ANR-12-VBDU-0002), and then used by the authors. These two days were previously defined by the participants of Trafipollu project. To validate this approach, the participants of Trafipollu project analyzed the variability of traffic and daily hourly profiles at Boulevard Alsace Lorraine on weekdays and weekdays. Observations regarding workdays were performed from 31 March 2014 to 04 April 2014, and those regarding weekends were performed at 29 and 30 March 2014, and 5, 6, 12 and 13 April 2014. Low variations of total traffic was observed during workdays (around $10 \%$ ). Slightly higher variations are observed on weekends (around $20 \%$ ), but the total traffic in these days are considerably lower than in workdays (approximately the half of total traffic in workdays). These low variations in total traffic validate the approach of using two representative days to simulate pollutant emissions. Other studies also mention the low variations in traffic emissions during the year, as the Source apportionment of airbone particles in the Île-de-France region, performed by the Air-quality agency of Paris (AIRPARIF, 2012).

Note that the Trafipollu web page is updated to https://anr.fr/ Projet-ANR-12-VBDU-0002.

11. Line 324: " $P M_{10}$ fraction" fraction of what? $P M_{10}$ fraction of TSP?

Author response: Yes. To improve clarity, the sentence 
$" f_{s}^{p m 10}$ the $P M_{10}$ fraction in each wear source $s "$

is replaced by

" $f_{s}^{p m 10}$ the $P M_{10}$ fraction of TSP emitted by each wear source $s "$.

12. Line 330: What is LCFs stand for by each letter?

Author response: It means load correction factor.

To improve clarity, the sentence

"They take into account the vehicle characteristics as HDV weight $\left(L C F_{s}\right)$ and the number of axles ( $\left.N_{\text {axle }}\right)$, as indicated in Equations (14) and (15)."

is replaced by

"They take into account the vehicle characteristics as HDV weight (represented by a load correction factor $\left.L C F_{s}\right)$ and the number of axles $\left(N_{a x l e}\right)$, as indicated in Equations (14) and (15)."

13. Section 4.1: Not clear if those 6 simulations are conducted based on Polairs3DMUNICH-SinG model combination or on a simple SinG local/box model.

Author response: All simulations in Section 4.1 were made using the SinG model. This model performs a two-way dynamic coupling between the regional-scale model Polair3D, and the local-scale model MUNICH, and can be seen as an interface between these models. With the two-way dynamic approach, the concentrations calculated in streets affect the concentrations calculated in the urban backgroud, and vice-versa.

Note that MUNICH can be also used as a stand-alone model, with a one-way coupling approach between regional and local scales. With this one-way coupling approach, 
the concentrations calculated in the streets do not affect the concentrations calculated in the urban background. The comparisons between the two-way ( $\operatorname{Sin} G$ ) and one-way (MUNICH as a stand-alone model) coupling approaches is performed in Section 5.

To improve clarity, the sentence

"Different simulations were performed, with large variations in tyre-wear emissions properties."

is replaced by

"Different simulations were performed using SinG, with large variations in tyre-wear emissions properties.".

14. Line 350: How dose those fractions applied for different size bins?

Author response: These fractions are used as constants, as indicated in the European Emission guidelines (Ntziachristos and Boulter, 2016).

To improve clarity, the sentence

"The $B C$ fraction adopted in brake and road wear are constant in all simulations, and follow the EMEP guidelines (Ntziachristos and Boulter, 2016) with $f_{b k}^{b c}=0.026$ and $f_{r d}^{b c}$ $=0.0106 . "$

is replaced by

"The BC fractions adopted in brake and road wear are constant in all simulations and they are the same in all emitted size sections. They follow the EMEP guidelines (Ntziachristos and Boulter, 2016) with $f_{b k}^{b c}=0.026$ and $f_{r d}^{b c}=0.0106$."

15. Table 1: Can you also add the model SOURCE in the table? Like EMEP, HERMES, 
Author response: Yes, it was added as footnote.

16: Line 395-398: I don't understand here. If the non-exhaust wear emissions are very low, there should be a very small amount of mass available for resuspension. So, no matter how high the resuspension rate is, there should have no mass supply for resuspension, as demonstrated by simulation 5 . So, how could a high resuspension rate along explained the much higher BC concentration in previous simulations? Isn't it just contradict your simulation 5 ?

Author response: The previous simulations mentioned in line 395 refer to Denby et al. (2013a) and Pay et al. (2011), not to simulations 2 and 3 of this study. Even if simulations 2 and 3 of this study use the same tyre, brake and road wear emission factors than those used by Pay et al. (2011) and Denby et al. (2013a), respectively, the parameterization employed to calculate particle resuspension are different. Pay et al. (2011) use constant resuspension emission factors, wich may not respect the mass balance over the street surface. Denby et al. (2013a) calculates the mass balance over the street surface, but artificially assuming that tyre, brake and road-wear emissions are instantly deposited over the street surface, and only these sources are employed to calculate the particle mass on the street. In both cases the authors found a relevant contribution of particle resuspension, and employed relatively low values of tyre-wear emission factors. Differently, in all simulations performed in this study, particle resuspension is computed by strictly respecting the mass balance over the street surface. Here the available mass at the street surface is computed based on particle deposition in the atmosphere, including all different sources, and particle wash-off, in rain episodes. With this approach, the particle resuspension calculated for black carbon does not strongly affect the concentrations in the street. These different approaches to calculate particle resuspension can explain the differences between the conclusions 
obtained here and those obtained by Pay et al. (2011) and Denby et al. (2013a), as discussed at lines 398-403.

To improve clarity, the sentence

"Previous simulations with the NORTRIP and HERMES models (Denby et al., 2013a; Pay et al., 2011) achieved good correlations between simulated and measured particle concentrations, because they assume that resuspension is the main non-exhaust emission process and use high resuspension rates."

is replaced by

"The simulations performed by Pay et al. (2011) and Denby et al. (2013a), using HERMES and NORTRIP models respectively, achieved good correlations between simulated and measured particle concentrations. They assume that resuspension is the main non-exhaust emission process, using high resuspension rates and relatively low tyre-wear emission factors.".

17. Line 442: Very confusing statement here, do you mean SinG and MUNICH are two different systems that can replace each other? But based on section 2, MUNICH is part of SinG. Or, maybe you want to say, the comparison is between Polair3D-SinGMUNICH and Polair3D-MUNICH (without SinG)? Same question for line 465.

Author response: The local-scale model MUNICH is part of SinG, but it can also be used as a stand-alone model. The different between these two approaches is the interactions between local (streets) and regional (urban background) scales.

As explained in lines 442-452: "the coupling between the local and regional scales is two way in SinG, which couple the street model MUNICH to the chemistry transport model Polair3D. However, MUNICH may be used as a standalone model, simulating the street concentrations with a one-way coupling to the regional-scale background concentrations. In that case, the regional-scale (background) concentrations influence 
the street concentrations, but the street concentrations do not influence the background concentrations, and the vertical mass transfer between local and regional scales only influences concentrations in the streets. Note that in the one-way coupling, traffic emissions are used both in the regional-scale model Polair3D and in the street model MU$\mathrm{NICH}$. Because SinG employs a two-way coupling, at each time step the vertical mass transfer between local and regional scales enables to calculate concentrations in the background and in streets, providing a direct interaction between concentrations in the street network and those in the urban background. Therefore, traffic emissions are considered only at the local scale, and there is no double counting of traffic emissions in SinG."

To improve clarity, these information are added and now briefly mentioned in Section 2. At line 204, the sentences

"As detailed in Lugon et al. (2020), this dynamic coupling between local and regional scales allows a direct interaction between concentrations in the street network and those in the urban background: the mass transfer between the street and the background concentrations influence both the street and the background concentrations. Furthermore, SinG uses consistent chemical and physical parameterizations, such as the same chemical module and meteorological data, at both local and regional scales."

are followed by

"Note that the street-network model MUNICH can also be used as a stand-alone model, with a one-way coupling approach. In this case the background concentrations influence the concentrations in the streets, but the mass transfer between streets and the background do not influence background concentrations.".

18. Line 444-448: What is the point to mention the one-way feedback here? Is it used in this study? Do you mean the Polair3D-SinG-MUNICH is using two-way feedback, 
and Polair3D-MUNICH is using one-way feedback? And you are comparing between those two methods?

Author response: Yes, exactly. This section compares the two approaches to calculate black carbon concentrations in the streets. Two simulations are performed using the same inlet data: $(i)$ one simulation uses $\operatorname{SinG}$, with the two-way coupling approach, and $(i i)$ the other simulation using $\mathrm{MUNICH}$ as a stand-alone model, with the one-way coupling approach.

To increase clarity, in addition to the notion of MUNICH used as a stand-alone model in Section 2 (please, see comment 17), the sentence

"This section investigates the influence of the two-way coupling between the regional and local scales on BC concentrations in the street network by comparing the concentrations simulated by SinG and MUNICH."

is replaced by

"This section investigates the influence of the two-way coupling between the regional and local scales on BC concentrations in the street network. For this, the concentrations calculated by $\operatorname{SinG}$, with the two-way approach, are compared with those calculated by MUNICH as a stand-alone model, with the one-way approach."

19. Line 469-470: Can you explain why this SinG results to higher BC concentrations compares to MUNICH for those streets?

Author response: As mentioned in line 470, these streets present high traffic emissions. According to the explanations of lines 456-462, "The vertical mass flux between the local and regional scales is proportional to the concentration gradient between the street and the urban background, as shown in Equation (8) of Lugon et al. (2020). Streets with high traffic emissions tend to favor the vertical mass transfer from the local to the regional scales, as they tend to present a high gradient between the street 
and the urban background concentrations. This vertical mass flux is also dependent of the street geometry, represented by the aspect ratio $\alpha r$ (see Equation 9 in Lugon et al. (2020)). Streets with low aspect ratio (large streets) tend to favor the vertical transfer between local and regional scales, and in streets with high aspect ratios (narrow streets) the vertical mass transfer between scales tends to be lower.

20. Line 477-478: How do this double-counting works? Please provide more detailed discussion here. Why does this not seeing on high emission street?

Author response: The double-counting concept is mentioned in lines 447-452, as follows:

"Note that in the one-way coupling, traffic emissions are used both in the regionalscale model Polair3D and in the street model MUNICH. Because SinG employs a twoway coupling, at each time step the vertical mass transfer between local and regional scales enables to calculate concentrations in the background and in streets, providing a direct interaction between concentrations in the street network and those in the urban background. Therefore, traffic emissions are considered only at the local scale, and there is no double counting of traffic emissions in SinG."

This approach is a simplified technique to represent the influence of the street network on background concentrations in the one-way coupling approach. Differently, with the two-way coupling approach, the mass transfer between streets and urban background is considered at both scales, and then there is no need to consider the traffic emissions at the regional scale. In streets with high traffic emissions, the concentration gradient favors the mass transfer between local and regional scales, and this mass flux is higher than the traffic emissions considered in the one-way coupling approach. In streets with low traffic emissions and high aspect ratios, it is the opposite: the vertical mass transfer between streets and urban background is low. The traffic emissions considered at the regional scale using the one-way coupling approach is then higher than the mass 
transfer between streets and urban background considered in the two-way coupling approach. In this case, this results in higher background concentrations above the streets using the one-way coupling approach, leading then to higher street concentrations.

To improve clarity, the sentence

"These large differences and the lower concentrations simulated in MUNICH compared to SinG may be explained by the double counting of traffic emissions performed by MUNICH with the one-way coupling technique."

is replaced by

"These large differences and the lower concentrations simulated in SinG compared to MUNICH may be explained by the double counting of traffic emissions performed by MUNICH with the one-way coupling technique. Using the two-way coupling approach in SinG, the streets with low traffic emissions and high aspect ratios present low vertical mass transfer between streets and urban background. This mass transfer is lower than the traffic emissions considered at the regional scale in the one-way coupling approach (MUNICH). This results in lower background concentrations above the streets using the two-way coupling approach (SinG), leading then to lower street concentrations. "

21. Section 5: Is the SinG simulation in Section 5 the same as simulation 4 in section 4 ? Is the MUNICH simulation in section 5 have the same model setup for all other SinG simulations? Why simulation result from the MUNICH simulation not compared with street-level observation (Boulevard Alsace Lorraine) as in Section 4? There is no way to judge which method has better model performance MUNICH or SinG.

Author response: Yes, the simulation using $\mathrm{MUNICH}$ as a stand-alone model (MUNICH-only) used the same input data as the simulation 4 presented in Section 4. Both MUNICH-only and SinG result very similar concentrations at Boulevard Alsace Lorraine, and they both respect the most strict performance criteria. The comparisons 
between $\mathrm{BC}$ concentrations observed at Boulevard Alsace Lorraine and those calculated with $\operatorname{SinG}$ and $\mathrm{MUNICH}$ are now included. The statistical indicators comparing SinG and MUNICH results are presented in the Appendix. For this, at line 453, the sentence

"Simulations of BC concentrations using Polair3D, MUNICH and SinG are performed using the non-exhaust emission factors of simulation 4 (see Table 1)."

is followed by

"Regarding specifically the BC concentrations at the Boulevard Alsace Lorraine, both SinG and MUNICH as a stand-alone model (MUNICH-only) resulted in similar concentrations, respecting the most strict performance criteria (see Table A1 in Appendix A2). However, the differences between MUNICH and SinG depend on the street characteristics, as detailed in Lugon et al (2020). To analyze streets with different characteristics, this section focus on the comparison between the one-way and two-way coupling approaches over the whole street network.".

And the following Table is included in the Appendix section:

Table 1. Comparisons to BC measurements at "Boulevard Alsace-Lorraine": statistical indicators obtained with SinG and MUNICH-only simulations.

\begin{tabular}{lcccccccc}
\hline & $\mathrm{o}\left[\mu \mathrm{g} \cdot \mathrm{m}^{-3}\right]$ & $\mathrm{s}\left[\mu \mathrm{g} \cdot \mathrm{m}^{-3}\right]$ & $\mathrm{FB}$ & $\mathrm{MG}$ & NMSE & VG & FAC2 & NAD \\
\hline SinG & 6.07 & 4.91 & -0.21 & 0.82 & 0.29 & 1.27 & 0.77 & 0.19 \\
MUNICH-only & 6.07 & 4.97 & -0.19 & 0.82 & 0.33 & 1.32 & 0.74 & 0.20 \\
\hline
\end{tabular}

